\title{
Pilot testing of a hydraulic bridge exciter
}

\author{
Andreas Andersson ${ }^{1,2}$, Mahir Ülker-Kaustell ${ }^{1,3, a}$, Richard Borg ${ }^{1}$, Olivier Dymén ${ }^{1}$, Anders Carolin $^{2}$, Raid Karoumi ${ }^{1}$ \\ ${ }^{1}$ Royal Institute of Technology, Division of Structural Engineering and Bridges, Stockholm, Sweden \\ ${ }^{2}$ The Swedish Transport Administration (Trafikverket), Sweden \\ ${ }^{3}$ Tyréns $A B$, Stockholm, Sweden
}

\begin{abstract}
This paper describes the development of a hydraulic bridge exciter and its first pilot testing on a full scale railway bridge in service. The exciter is based on a hydraulic load cylinder with a capacity of $50 \mathrm{kN}$ and is intended for controlled dynamic loading up to at least $50 \mathrm{~Hz}$. The load is applied from underneath the bridge, enabling testing while the railway line is in service. The system is shown to produce constant load amplitude even at resonance. The exciter is used to experimentally determine frequency response functions at all sensor locations, which serve as valuable input for model updating and verification. An FE-model of the case study bridge has been developed that is in good agreement with the experimental results.
\end{abstract}

\section{Introduction}

Within the research field of bridge dynamics, in-situ field measurements of existing structures often serve as a vital input in both understanding the real structural manner of action and updating mathematical models. Properties such as natural frequencies, mode shapes and damping ratios are commonly estimated. Experimental testing can be divided in two classes; input-output methods and output-only methods. A known input may consist of an instrumented impact hammer or a load shaker for continuous forced vibration. The output-only methods, such as the stochastic subspace identification, are highly efficient for linear time-invariant systems [1]. For large structures as bridges or high-rise buildings, assuming background vibrations or wind excitation as timeinvariant often produce successful results $[2,3]$. For some structures however, an amplitude dependent response may be observed due to nonlinearities. A category of such structures are railway bridges, where ambient vibrations from wind loads typically are much less than the response from passing trains. Research has shown that bearing frictions and interaction with the ballasted railway track are potential sources of nonlinearities [4]. Calibrating mathematical models based on ambient vibrations may therefore fail to accurately predict the real response from passing trains. This has gained increased attention in recent years, especially when upgrading existing railway lines to higher speeds $[5,6]$. When using the high-amplitude response induced by a passing train, challenges arise with train-bridge interaction and the short duration of the excitation.

To overcome some of these challenges, a hydraulic bridge exciter has been developed. The aim is to subject the bridge to a continuous forced vibration of constant amplitude and with sufficient magnitude to study relevant effects of nonlinearities. This paper describes the very first pilot testing using this device.

\section{Exciter design}

There are several examples of devices for controlled dynamic excitation of bridges, e.g. $[7,8,9,10]$. They are often based on either an electrodynamic shaker that enables a constant amplitude input force or a fixed rotating mass device where the input force is proportional to the square of the angular velocity of the rotating mass. Both categories involve fairly heavy equipment, both to withstand the dynamic loads but also to avoid uplift due to the mass inertia. The devices are often placed directly on the bridge deck for road bridges or on a trolley for railway bridges. For railway bridges on existing lines, access to the track area normally involves long planning even for only a few hours during night.

With the above in mind, an exciter has been developed based on a hydraulic load cylinder that is prestressed from the ground to the bridge soffit. The intended application is short to medium span railway bridges passing over land or roads. The vertical clearance for the underlying road is typically $4.5-6 \mathrm{~m}$. This distance is reached using a vertical truss column. The schematics of the exciter are illustrated in Figure 1.

The hydraulic cylinder has a capacity of $50 \mathrm{kN}$ which is deemed sufficient compared to a typical railroad axle load of some $200 \mathrm{kN}$. The target frequency range is $0-$ $50 \mathrm{~Hz}$ but can be increased for structures of small displacement magnitudes.

The ground support consists of a $0.9 \times 0.9 \mathrm{~m}$ square plate, onto which the hydraulic cylinder is mounted. To

\footnotetext{
${ }^{\mathrm{a}}$ Corresponding author: Mahir.Ulker-Kaustell@tyrens.se
} 
enable an easy adjustment of the total height, a set of VKR $220 \times 220 \times 6.3$ beam sections can be stacked in between the cylinder and the plate. The lower end of the truss is connected to the piston of the cylinder and the upper end to the bridge deck. The truss is built in segments to enable variable heights and to make manual handling and transportation easier. Hinges are located at the lower end of the cylinder and at the connection to the bridge. For increased stability, four diagonal M16 bars connect from the ground plate to the upper end of the cylinder. The input force is measured with a load cell at the top of the truss. The hydraulic cylinder is powered by an external diesel driven oil pump system. The load cell, the piston displacement and the oil pressure is connected to an MTS FlexTest SE controller that assures a constant amplitude input force. The controller is able to account for any flexibility of the ground and the truss. Details of the lower and upper connections of the bridge exciter are shown in Figure 2.

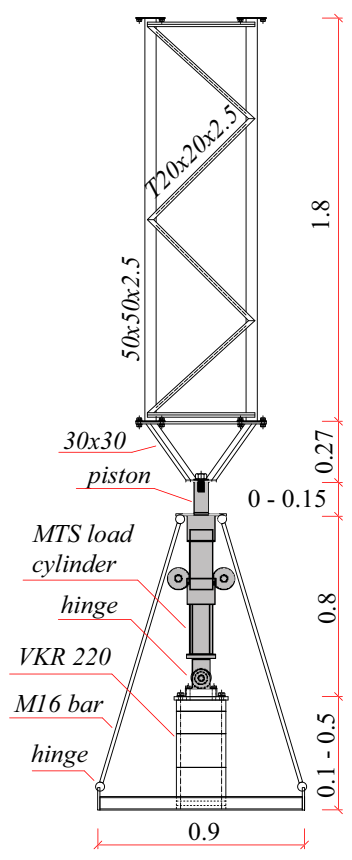

a)

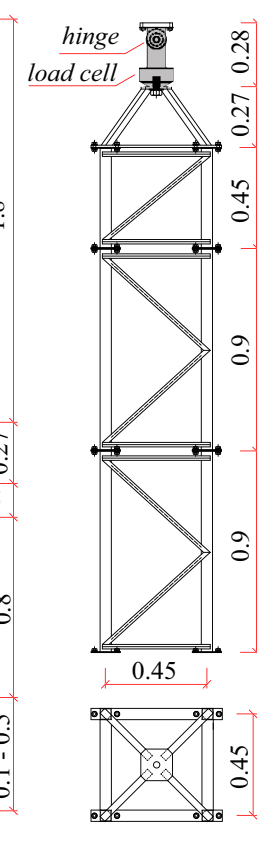

(1)

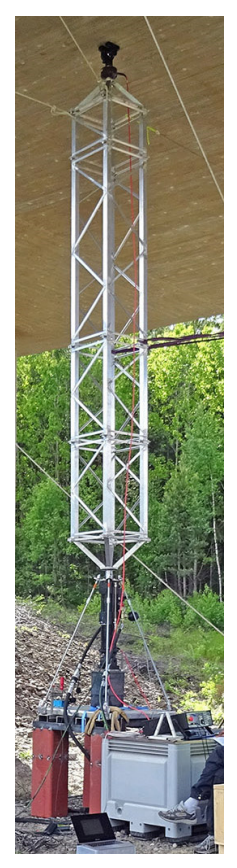

b)
Figure 1. The hydraulic bridge exciter, a) schematics, b) photo when mounted on the case study bridge.

To enable manual handling of all components, the truss and the ground plate are made of aluminium. The system was optimized to assure a sufficient ultimate load capacity including buckling, fatigue strength and to avoid natural frequencies within the target frequency range of $50 \mathrm{~Hz}$. The system is intended to work in compression and therefore relies on a static pre-load. Hence, the peak load amplitude is $25 \mathrm{kN}$.

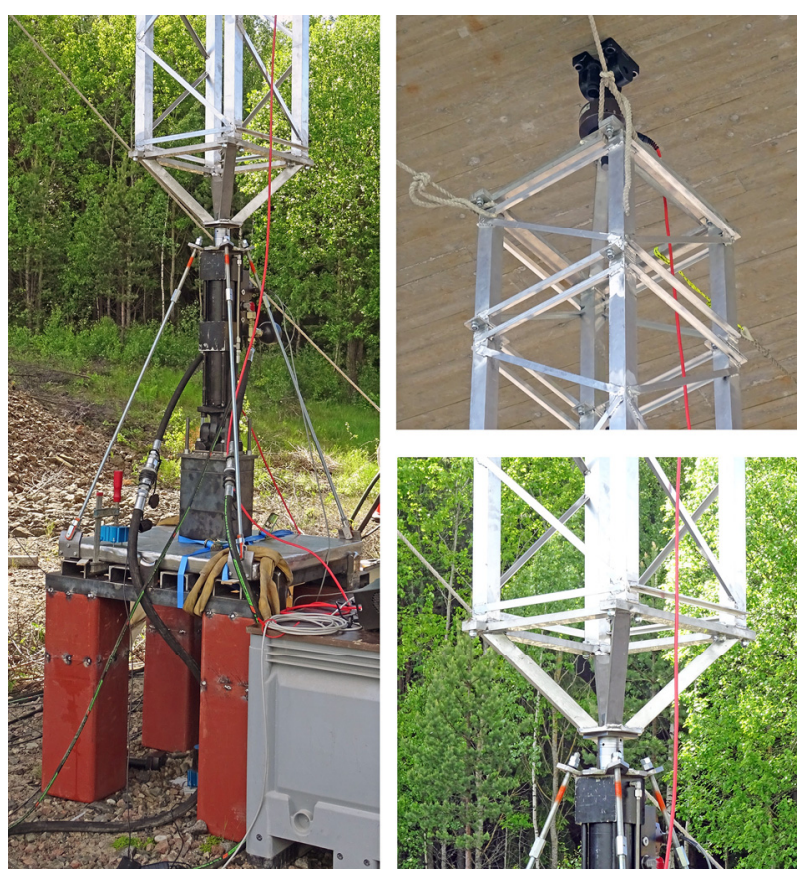

Figure 2. Details of the Bridge exciter.

\section{Case study Bridge}

The pilot tests were performed on a three span railway bridge in Södertälje, $35 \mathrm{~km}$ South-West of Stockholm, Sweden. The bridge carries two ballasted tracks and is designed as a continuous concrete slab bridge. A photo of the bridge is shown in Figure 3.

The bridge was previously included in a feasibility study with the aim of increasing the speed on existing railway lines [6]. Despite the large inherent mass of the structure, a 2D analysis predicted excessive vibrations during train passages. The reason was believed to be due to the large over-sail at the end supports, see Figure 4. Within an ongoing research project, similar structures are studied, focusing on the dynamic response and the interaction with the surrounding backfill.

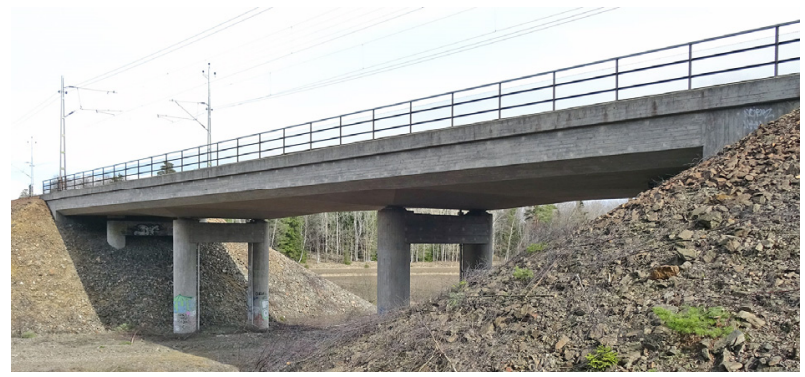

Figure 3. Photo of the case study bridge.

\subsection{Bridge properties}

The geometry of the bridge is presented in Figure 4. The main span is $18.4 \mathrm{~m}$ and the side spans $11.1 \mathrm{~m}$. The oversail is $3.0 \mathrm{~m}$, onto which an integral structure consisting of back walls and wing walls are connected. The bridge deck is supported by the columns using fixed bearings $(f b)$ in the main span and sliding bearings $(s b)$ at the end 
supports. The integral structure interacts with the surrounding embankment and track structure.

\subsection{Instrumentation and testing}

The experimental tests were performed on June 3, 2015. Accelerometers (uni-axial SiFlex SF1500S) were mounted on top of the edge beam, denoted $a_{1}$ to $a_{12}$ in Figure 4. Due to time constraints and accessibility, sensors $a_{4}, a_{5}$ and $a_{11}$ were never installed and sensor $a_{8}$ was not placed parallel to $a_{2}$. Data was collected using a HBM MGCPlus data acquisition system and a sample frequency of $600 \mathrm{~Hz}$. A total of four tests were performed, denoted test 1 to test 4 in Table 1 . The tests were performed with regular traffic in service, resulting in a few passing trains interfering with the experimental testing. The response from the trains was however successfully mitigated by using a narrow band-pass filter, centred at the loading frequency during the train passage.

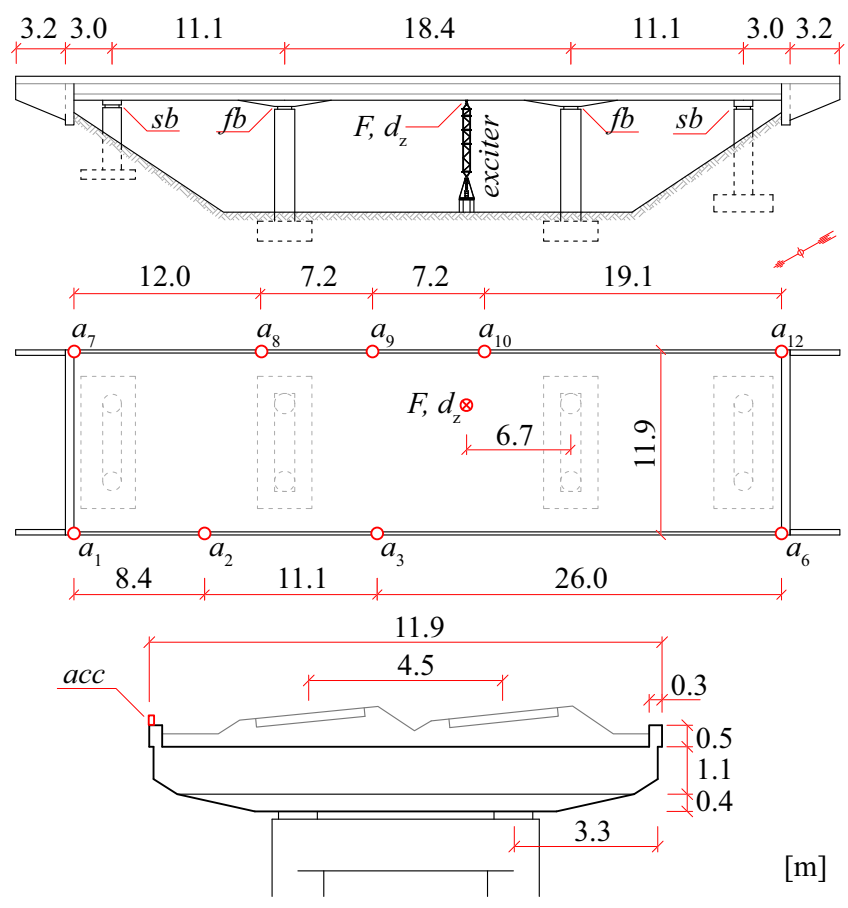

Figure 4. Drawing of the bridge illustrating the geometry and sensor positions.

Table 1. Settings for the hydraulic exciter during the tests.

\begin{tabular}{|c|c|c|c|c|}
\hline Test: & $\begin{array}{c}\text { Pre-load } \\
(\mathbf{k N})\end{array}$ & $\begin{array}{c}\text { Amplitude } \\
(\mathbf{k N})\end{array}$ & $\begin{array}{c}\text { Range } \\
(\mathbf{H z})\end{array}$ & $\begin{array}{c}\text { Rate } \\
(\mathbf{H z} / \mathbf{s})\end{array}$ \\
\hline test1 & 10 & 5 & $3-20-3$ & 0.10 \\
\hline test2 & 10 & 5 & $3-50$ & 0.05 \\
\hline test3 & 15 & 10 & $3-50$ & 0.05 \\
\hline test4 & 15 & 10 & $5-10$ & 0.01 \\
\hline
\end{tabular}

\section{Experimental results}

\subsection{Time domain response}

An example of the measured time response is shown in Figure 5. The system is able to produce a constant amplitude input load, even at resonance. The displacement at the hydraulic cylinder is about $1 \mathrm{~mm}$ and includes both the displacement of the bridge and the flexibility of the supports under the bridge exciter. The load amplitude of $10 \mathrm{kN}$ results in a maximum acceleration of about $0.6 \mathrm{~m} / \mathrm{s}^{2}$ at sensor $a_{10}$. The system has a load amplitude capacity of $25 \mathrm{kN}$, potentially resulting in a peak acceleration of $1.5 \mathrm{~m} / \mathrm{s}^{2}$. It was however decided not to test the peak load capacity during the pilot tests.

The dynamic behaviour of the bridge is rather complicated, consisting of both bending, torsional and plate modes. In this paper, the three modes with the largest contribution are further studied, denoted mode 1 to mode 3 , although intermediate modes may exist.
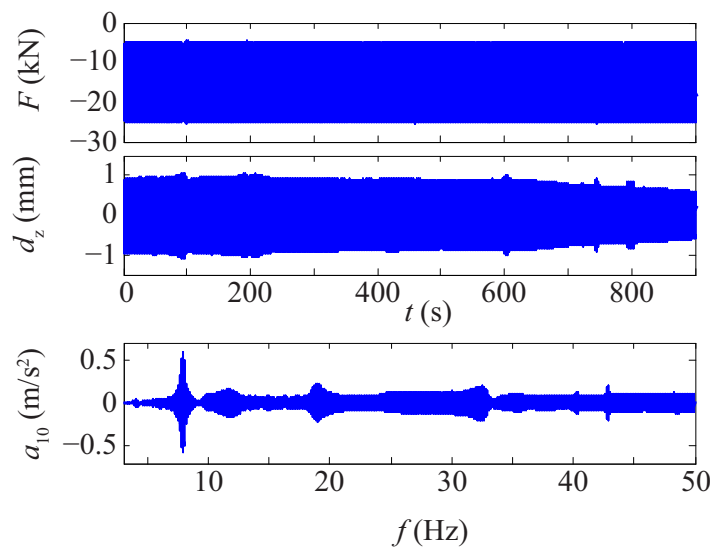

Figure 5. Load and vertical displacement from the exciter, vertical acceleration at sensor $a_{10}$ from test 3 .

A first modal identification is performed by studying the phase shift between sensor $a_{3}, a_{9}$ and $a_{10}$ in the main span, Figure 6. The first mode at $7.8 \mathrm{~Hz}$ is likely a vertical bending mode, since all three sensors are in phase and of similar magnitude. The second mode at $11.6 \mathrm{~Hz}$ is likely a torsional mode since sensor $a_{3}$ is out of phase with sensor $a_{9}$ and $a_{10}$ on the opposite side of the bridge. The third mode at $18.9 \mathrm{~Hz}$ is likely a second bending mode, since sensor $a_{10}$ is out of phase compared to sensor $a_{3}$ and $a_{9}$.
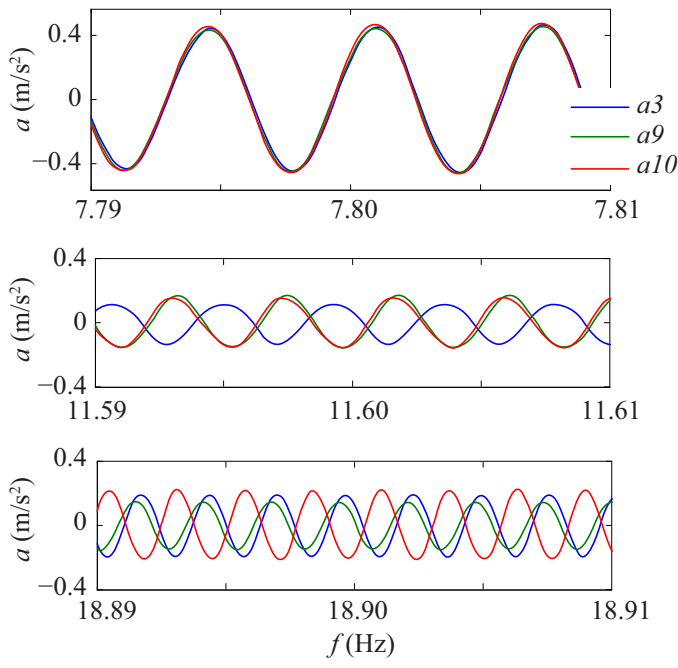

Figure 6. Time response at sensor $a_{3}, a_{9}$ and $a_{10}$ at the first three Eigen-modes. 
The mode shapes are further estimated by plotting the acceleration vs. the sensor location, as illustrated in Figure 7. A spline interpolation has been used between the sensor locations (circles) and assuming zero response at the support. This is not a completely accurate assumption, since the supports are located about $3.3 \mathrm{~m}$ inward from the edge beam, shown in the lower Figure 4. Due to the limited number of sensors, estimating higher order modes are afflicted with increasing uncertainties. As an example, the third mode is likely a vertical bending mode but may be mistaken for a torsional mode since sensor $a_{4}$ was never installed opposite to sensor $a_{10}$. It is also worth noting the movement of the bridge end points.

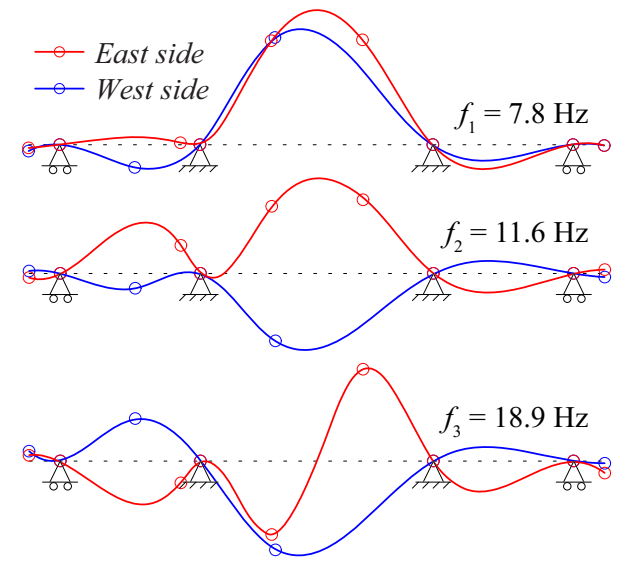

Figure 7. Mode shape estimation using the measured vertical acceleration from test 3 and spline-fitting.

\subsection{Frequency domain response}

Since the bridge was excited with a known input load of constant amplitude, the frequency response function (FRF) is obtained simply by dividing measured acceleration with the load amplitude. To enable a comparison between the four tests, all results have been scaled to a $10 \mathrm{kN}$ load amplitude. The signals from test 2 contain slightly more noise due to some gaps between the ground plate and the support structure of the hydraulic shaker. This was later tightened and test 3 and test 4 were running more smoothly.

The FRF at sensor $a_{1}$ and $a_{10}$ is shown in Figure 8, showing the range for the first mode. It is observed that the resonance frequency is slightly lower in test 3 and test 4 , potentially owing to the increased load amplitude. The FRF at sensor $a_{10}$ is also shown in Figure 9 for the range up to $40 \mathrm{~Hz}$.

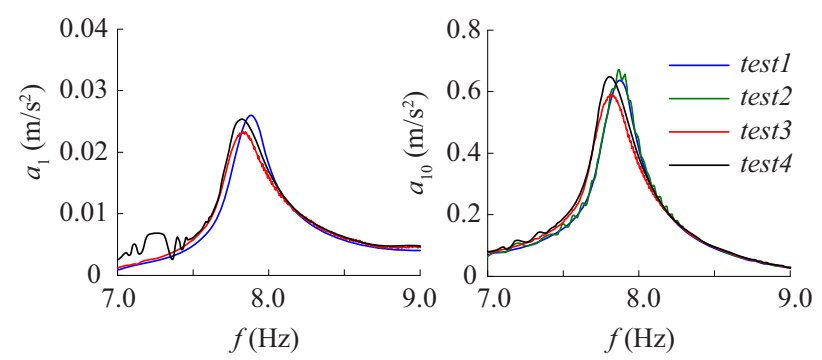

Figure 8. FRF of the first mode at $7.8 \mathrm{~Hz}$, sensor $a_{1}$ and $a_{10}$.

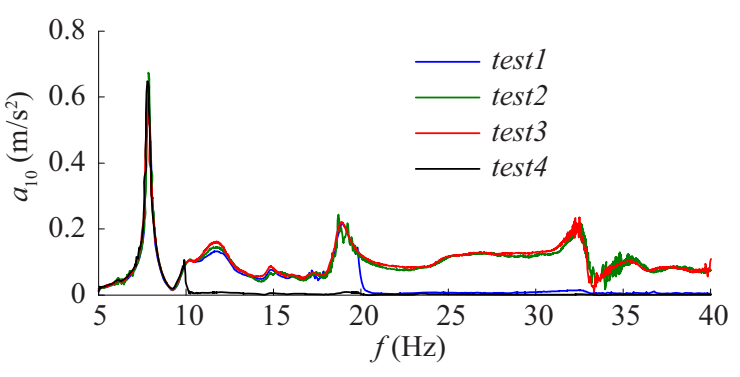

Figure 9. FRF within the range $5-40 \mathrm{~Hz}$, sensor $a_{10}$.

\subsection{Damping estimates}

The damping ratio for the three studied modes was estimated using the Half-Power Bandwidth method in frequency domain, Equation (1), where $f_{\mathrm{a}}$ and $f_{\mathrm{b}}$ are found at $a_{\max } / \sqrt{2}$. The results from test 3 are shown in Figure 10. For a yet unknown reason, the torsional mode at $11.6 \mathrm{~Hz}$ has a significantly larger damping compared to the first and third mode. The results are rather consistent for all tests, as presented in Table 2.

$$
\zeta=\frac{f_{\mathrm{b}}-f_{\mathrm{a}}}{f_{\mathrm{a}}+f_{\mathrm{b}}}
$$
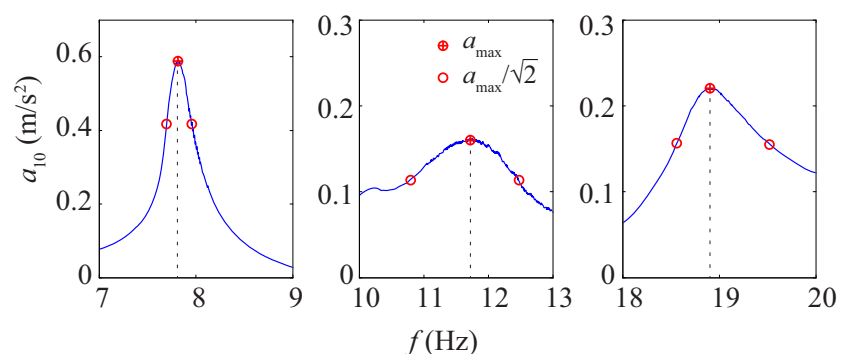

Figure 10. Damping estimates of the three first modes using the Half-Power Bandwidth method, based on sensor $a_{10}$ from test3.

Table 2. Estimated natural frequencies and damping ratio.

\begin{tabular}{|c|c|c|c|c|c|c|}
\hline & $\boldsymbol{f}_{\mathbf{1}}(\mathrm{Hz})$ & $\boldsymbol{\zeta}_{\mathbf{1}}(\mathbf{\%})$ & $\boldsymbol{f}_{\mathbf{2}}(\mathrm{Hz})$ & $\boldsymbol{\zeta}_{\mathbf{2}}(\boldsymbol{\%})$ & $\boldsymbol{f}_{\mathbf{3}}(\mathrm{Hz})$ & $\boldsymbol{\zeta}_{\mathbf{3}}(\mathbf{\%})$ \\
\hline test1 & 7.87 & 1.4 & 11.62 & - & 18.91 & 2.4 \\
\hline test2 & 7.82 & 1.6 & 11.60 & 9.3 & 18.90 & 2.3 \\
\hline test3 & 7.80 & 1.7 & 11.60 & 7.4 & 18.90 & 2.5 \\
\hline test4 & 7.81 & 1.6 & - & - & - & - \\
\hline
\end{tabular}

\section{Numerical simulations}

One of the main purposes of performing the tests with the hydraulic exciter is to use the results in calibrating and verifying theoretical models. For the purpose of the case study bridge, a 3D FE-model has been developed.

\subsection{The FE-model}

The FE-model of the bridge is depicted in Figure 11. The bridge deck, the back-walls and the wing walls are modelled with 4-noded shell elements. The deck thickness is $1.1 \mathrm{~m}$ which increases to $1.5 \mathrm{~m}$ over the 
supports. The mass of the ballasted track is included by increasing the density of the bridge deck.

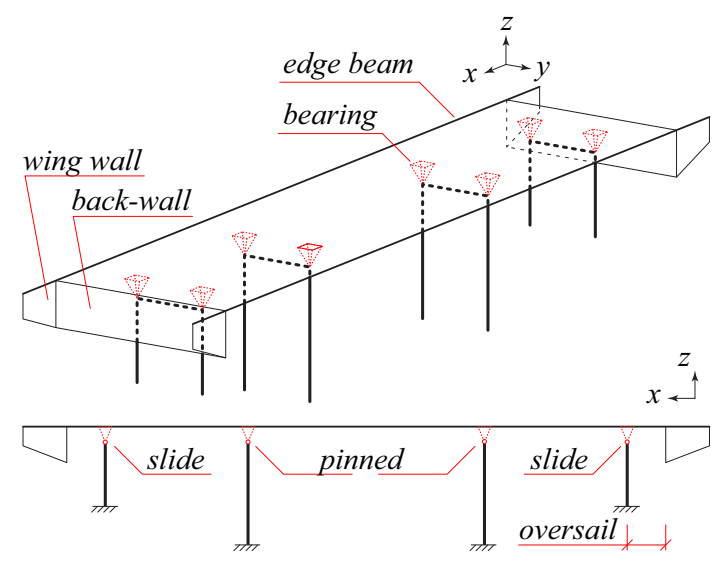

Figure 11. View of the FE-model.

The concrete is assumed to have a density of $2400 \mathrm{~kg} / \mathrm{m}^{3}$ and a Young's modulus of $34 \mathrm{GPa}$.

The edge beams have a rectangular section of $0.3 \times 0.5 \mathrm{~m}$ and are modelled as beam elements from each end of the bridge and rigidly connected to the shell elements. The columns are circular with a diameter of $1.3 \mathrm{~m}$ and the connecting cross-beams have a square section of $1.2 \times 1.2 \mathrm{~m}$. Both the columns and the crossbeams are modelled with beam elements. The lower part of the columns is assumed fully fixed to the ground. The upper part of the columns is connected to the bridge deck via constraint equations, acting as a pinned support at the mid columns and enabling sliding in the longitudinal direction at the end supports.

The interaction between backfill and the adjacent back-wall and wing walls are modelled with solid elements (not illustrated). The interface between the soil and the concrete is assumed not to enable sliding under current load amplitudes, hence acting fully linear. The soil is assumed to have a Young's modulus of $200 \mathrm{MPa}$.

\subsection{Eigenvalue analysis}

An eigenvalue analysis of the FE-model results in about 30 modes within the range $6-30 \mathrm{~Hz}$. Many of the modes are dominated by lateral components that will not be excited from either passing trains or the hydraulic exciter. In Figure 12, the three lowest vertical modes are presented, showing great similarities with the experimental results.

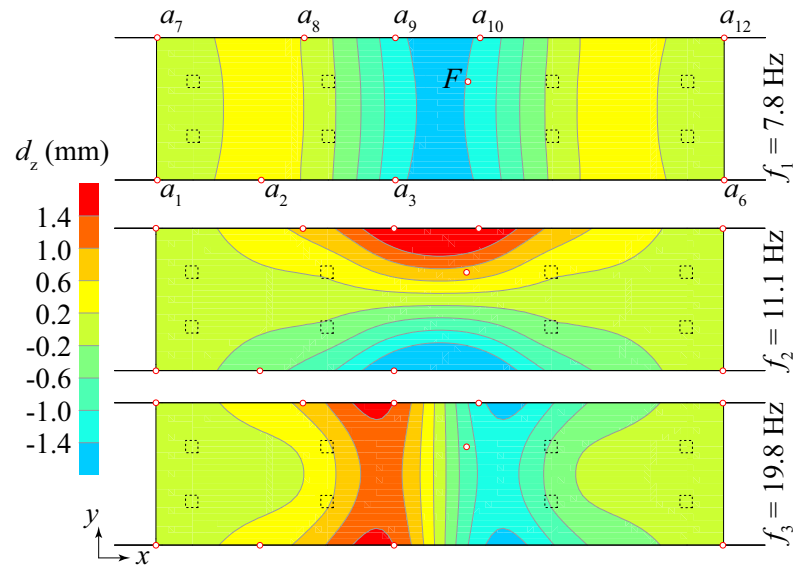

Figure 12. Eigenvalue analysis showing the first three modes.

\subsection{Comparison with experimental results}

A set of FRFs are calculated from the FE-model at similar locations as the sensors from the experiment. Firstly, the damping matrix $\mathbf{C}$ is estimated as a linear combination of the mass matrix $\mathbf{M}$ and the stiffness matrix K, commonly known as Rayleigh damping, Equation (2). The constants $\alpha$ and $\beta$ are obtained according to Equation (3). In the present case, $f_{\mathrm{n}}=$ $7.8 \mathrm{~Hz}, \zeta_{\mathrm{n}}=1.7 \%$ and $f_{\mathrm{m}}=18.9 \mathrm{~Hz}, \zeta_{\mathrm{m}}=2.5 \%$. As a result, the damping at the second mode will be underestimated.

$$
\mathbf{C}=\alpha \mathbf{M}+\beta \mathbf{K}
$$

$$
\begin{aligned}
& \alpha=2 \omega_{\mathrm{n}} \omega_{\mathrm{m}}\left(\zeta_{\mathrm{n}} \omega_{\mathrm{n}}-\zeta_{\mathrm{m}} \omega_{\mathrm{m}}\right) /\left(\omega_{\mathrm{n}}{ }^{2}-\omega_{\mathrm{m}}{ }^{2}\right) \\
& \beta=2 \omega_{\mathrm{n}} \omega_{\mathrm{m}}\left(-\zeta_{\mathrm{n}} / \omega_{\mathrm{n}}+\zeta_{\mathrm{m}} / \omega_{\mathrm{m}}\right) /\left(\omega_{\mathrm{n}}{ }^{2}-\omega_{\mathrm{m}}{ }^{2}\right)
\end{aligned}
$$

The steady-state response can be obtained by solving the equation of motion in Equation (4) for the frequency dependent displacement vector $x(\omega)$. The load vector $\mathbf{F}$ consists of a constant stationary load at the point where the bridge exciter is located.

$$
\left(-\omega^{2} \mathbf{M}+i \omega \mathbf{C}+\mathbf{K}\right) x(\omega)=\mathbf{F}
$$

The resulting FRF at location $a_{10}$ is shown in Figure 12. As expected, the FE-model predicts a larger response at the second mode due to the lower damping. The third mode is of similar magnitude and shape of the FRF, although slightly shifted in frequency.

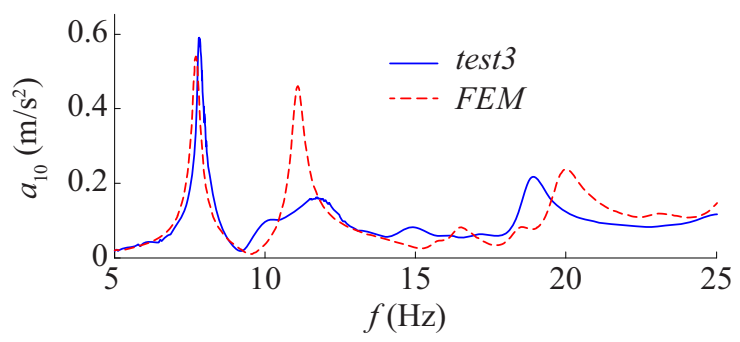

Figure 13. FRF of sensor $a_{10}$, comparison between experiments during test 3 and the FE-model. 


\section{Conclusions}

From the presented pilot tests of the hydraulic bridge exciter on the case study bridge, the following is concluded.

- The bridge exciter was shown to produce a stable constant amplitude load, even at resonance.

- Frequency response functions have been determined experimentally with high accuracy using a linear frequency sweep.

- The bridge exciter was able to generate a vertical deck acceleration of about $0.6 \mathrm{~m} / \mathrm{s}^{2}$, with a potential maximum of $1.5 \mathrm{~m} / \mathrm{s}^{2}$. This is deemed rather significant considering that the case study bridge was a three-span, two track railway bridge.

- The experimental results are in good agreement with a numerical model.

- Using the full frequency response functions serve as a valuable input in model updating and will enable improved accuracy in dynamic assessments of existing bridges.

\section{Acknowledgements}

The development of the hydraulic bridge exciter has been funded by the Swedish Transport Administration (Trafikverket). The laboratory technicians $\mathrm{Mr}$. Claes Kullberg, Mr. Stefan Trillkott and Mr. Kent Lindgren have contributed with invaluable support in the practical development of the bridge exciter and knowledge in hydraulic systems and experimental testing.

\section{References}

1. R. Brincker, P. Andersen, SVIBS, 2 (2006).

2. C. E. Ventura, R. Brincker, E. Dascotte, P. Andersen, IMAC XIX (2001), pp. 324-330.

3. F. Magalhães, E. Caetano, Á. Cunha, ASCE Bridge Eng., 12, 6 (2007), pp. 746-754.

4. M. Ülker-Kaustell, R. Karoumi, IJRT 1, 4 (2013), pp. 237-257.

5. C. Johansson, N. Nualláin, C. Pacoste, A. Andersson, Eng. Struct 58 (2014), pp. 25-35.

6. C. Johansson, T. Arvidsson, D. Martino, M. Solat, A. Andersson, C. Pacoste, R. Karoumi, ISSN 11034289, ISRN KTH/BKN/R-141-SE (2011).

7. N. Haritos, H. Khalaf, T. Chalko, IMAC XIII (1995), pp. $703-709$.

8. M. Link, R. G. Rohrmann, S. Pietrzko, IMAC XIV (1996), pp. $218-225$.

9. J. Swolski, J. Bien, J. of Civ. Eng. \& Management 17, 4 (2011), pp. 590-599.

10. P. Omenzetter, S. Beskhyroun, F. Shabbir, G-W. Chen, X. Chen, S. Wang, A. Zha, Earthquake Commission Research UNI/578 (2013). 